\title{
Critical Care After Lung Resection (CALOR) - Influence of Anaesthetic and Analgesic Technique
}

I. Orzechowska ${ }^{1}$, P. M. Mccall2 , A. Glass' ${ }^{2}$ A. Klein ${ }^{3}$, B. Shelley², on behalf of the ACTACC CALoR-2 collaborators

${ }^{1}$ London School of Hygiene and Tropical Medicine - London (United Kingdom), ${ }^{2}$ University of Glasgow - Glasgow (United Kingdom), ${ }^{3}$ Papworth Hospital - Cambridge (United Kingdom)

\section{INTRODUCTION}

Un-planned critical care admission is a devastating complication of lung resection, associated with increased mortality and additional burden on healthcare services [1]. Existing data indicate that 2.8-18\% of patients require unplanned intensive care following pulmonary resection [2]. Within the UK Association of Cardiothoracic Anaesthesia and Critical Care's annual collaborative audit into Critical Care Admission following Lung Resection (CALoR) we sought to investigate the influence of anaesthetic and analgesic techniques on the need for unplanned post-operative critical care.

\section{DATA}

This was a multicentre retrospective audit examining adult patients undergoing lung resection in the years 2013-2014. Demographic and clinical data was collected on every patient fulfilling *inclusion criteria. Control data was collected from a minimum of three consecutive contemporaneous control patients undergoing surgery prior to each case.

Un-planned critical care admission formally defined as:

- patient required intubation for invasive ventilation and/or

- renal replacement therapy (RRT) post-operatively;

- unplanned mechanical ventilation immediately postoperatively for greater than 12 hours

\section{METHODS}

Association was sought between mode of anaesthesia (total intravenous anaesthesia (TIVA) vs volatile) and analgesic technique (epidural vs paravertebral) and the need for critical care admission. Multivariate regression analyses were performed on a 'complete record' basis for each variable. A sensitivity analysis was conducted as both variables of interest had over $20 \%$ missing values. We devised an imputation model using multiple imputation by chained equations and estimates were compared with those from the complete-record model.

RESULTS

Data was returned by sixteen centres. Sufficient data for analysis was available from 7,431 patients; 7178 controls and 253 cases. The overall incidence of unplanned ICU admission was $2.26 \%(n=253)$.

Analgesia was provided by epidural in $26.9 \%$ and paravertebral blockade in $41.8 \% ; 77.2 \%$ of cases received volatile anaesthesia. Across univariate, complete-case and multiple imputation models there was a strong and significant effect of both anaesthetic and analgesic technique on need for critical care admission. Patients receiving TIVA were estimated to have approximately half the odds of critical care admission compared to these undergoing volatile anaesthesia, whilst patients receiving epidural analgesia had just over half the odds of admission compared to those receiving paravertebral (Table 1).

Table 1. Association Between Anaesthetic and Analgesic technique and critical care admission

\begin{tabular}{|c|c|c|c|c|c|c|c|c|c|}
\hline \multirow[t]{2}{*}{ Exposure } & \multicolumn{3}{|c|}{ Univariate } & \multicolumn{4}{|c|}{ Complete-record model* } & \multicolumn{2}{|c|}{ Imputation model* } \\
\hline & $\mathbf{N}$ & OR & $95 \% \mathrm{Cl}$ & $\mathbf{N}$ & OR & $95 \% \mathrm{Cl}$ & $\mathbf{N}$ & OR & $95 \% \mathrm{Cl}$ \\
\hline $\begin{array}{l}\text { Anaesthetic } \\
\text { technique }\end{array}$ & 4,318 & & & 3,970 & & & 7,401 & & \\
\hline Volatile & & 1.00 & & & 1.00 & & & 1.00 & \\
\hline TIVA & & 0.59 & $0.41-0.84$ & & 0.45 & $0.32-0.65$ & & 0.50 & $0.34-0.69$ \\
\hline $\begin{array}{l}\text { Analgesic } \\
\text { Technique }\end{array}$ & 3,683 & & & 3,668 & & & 7,431 & & \\
\hline PVB & & 1.00 & & & 1.00 & & & 1.00 & \\
\hline Epidural & & 0.77 & $0.57-1.05$ & & 0.57 & $0.41-0.78$ & & 0.57 & $0.41-0.79$ \\
\hline
\end{tabular}

*adjusted for age, sex, resection type, surgical approach

- This large retrospective study suggests a significant effect of both anaesthetic and analgesic technique on patient outcome.

- The results in favour of both TIVA and epiduralanalgesia might be considered to conflict with recent studies.

- We suggest that as a community of thoracic anaesthetists, it is incumbent on us all to attempt to address these fundamental questions in well conducted, large-scale randomised controlled trials.

\section{REFERENCES}

\{1].Pedoto, Alessia, and Paul M. Heerdt. "Postoperative care after pulmonary resection: postanesthesia care unit versus intensive care unit." Current Opinion in Anesthesiology 22.1 (2009): 50-55

[2] Brunelli, Alessandro, et al. "A scoring system predicting the risk for intensive care unit admission for complications after major lung resection: a multicenter analysis." The Annals of thoracic surgery 86.1 (2008): 213-218. 